\title{
Filigrane
}

Écoutes psychothérapiques

\section{Présentation. Psychanalyse et temporalités, deuxième partie}

\section{Sophie Gilbert et Véronique Lussier}

Volume 22, numéro 2, automne 2013

Psychanalyse et temporalités II

URI : https://id.erudit.org/iderudit/1022552ar

DOI : https://doi.org/10.7202/1022552ar

Aller au sommaire du numéro

Éditeur(s)

Revue Santé mentale au Québec

ISSN

1192-1412 (imprimé)

1911-4656 (numérique)

Découvrir la revue

Citer ce document

Gilbert, S. \& Lussier, V. (2013). Présentation. Psychanalyse et temporalités, deuxième partie. Filigrane, 22(2), 7-8. https://doi.org/10.7202/1022552ar d'utilisation que vous pouvez consulter en ligne. 


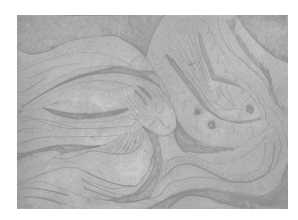

\section{Présentation. Psychanalyse et temporalités, deuxième partie}

\section{Sophie Gilbert et Véronique Lussier}

ette seconde partie de notre dossier sur les temporalités en psychanalyse nous amène à poursuivre l'exploration de ce vaste concept, cette fois sous l'angle particulier de l'arrimage entre temps et espace: autres contrées et autres époques, mais aussi la prise en compte de la situation analytique comme lieu de rencontre. Dans ce numéro, des considérations historiques et culturelles sont élaborées, en parallèle avec la perspective du fonctionnement psychique, dans la normalité comme dans la pathologie. Le lecteur ne pourra que constater combien la psychanalyse nous propose un temps autre, qui alimente encore aujourd'hui une pratique créatrice et fertile. Sous des angles complémentaires, nos auteurs rappellent aussi combien la psychanalyse est indissociable de la notion du lien social. Que celui-ci soit abordé dans une perspective de transmission générationnelle, de confrontation à une altérité menaçante, ou de relation transférentielle.

Notre dossier s'amorce avec une riche contribution de Janine Altounian. Reconnue pour ses traductions de l'œuvre de Freud, l'auteure nous présente ici l'arrimage du temps psychique, généalogique et historique dans la publication d'une œuvre qui lui fut léguée, soit un témoignage personnel sur les traumas infligés par la déportation du peuple arménien. Janine Altounian nous autorise à la suivre sur le chemin sinueux des enjeux personnels et sociaux du traumatisme, qui croise celui d'un travail itératif de transmission adressée à l'autre: l'autre génération, mais aussi l'autre culture.

Puis, dans un registre plus clinique et sous un tout autre angle, ce thème de la violence telle qu'articulée à la temporalité psychique est abordé par Philippe Jeammet. Ici, la destructivité intrinsèque au sujet humain est considérée comme deuxième temps, ou même une possible brèche dans la linéarité d'un temps partagé. Il s'agit pour l'auteur de défendre la proposition de la destruction comme tentative de rétablir seul et pour soi une homéostasie 
perdue, ou de réagir à une menace de perturbation de celle-ci. La violence alimente ainsi des symptômes qui - bien que de façon malhabile - expriment néanmoins une volonté de vie.

Dans un registre didactique, Bernard Chervet propose aux lecteurs une étude extensive de la temporalité particulière de l'après-coup, là où la chronologie semble s'inverser, et dévoile non seulement l'intemporalité mais aussi, le hors temps. Après un bref détour historique, l'auteur nous fait progressivement la démonstration de la prévalence de ce processus dans le fonctionnement psychique et surtout, illustre les différentes modalités de son approche (incontournable) dans la situation clinique.

Pour clore ce dossier, Antonino Ferro et Giuseppe Civitarese nous proposent de revisiter Bion, par le biais du concept d' «espacement» - ou espace-temps. Les auteurs démontrent de façon théorique, puis illustrent cliniquement ce concept, en lien avec la notion de «champ analytique», un concept dont Ferro a particulièrement bien documenté la pertinence dans ses écrits précédents. Cette centralité de l'espacement dans la situation analytique convie l'analyste à une place singulière, où la connotation affective, voire émotionnelle du partage de cet espace-temps apparait fondamentale.

À la suite de ce dossier, la rubrique Héréros est l'occasion de compléter ce numéro en mettant l'accent cette fois sur l'autre extrémité de la polarité temps-espace. Les deux articles suivants abordent la question du lieu et plus précisément, la première et la troisième topique, en référence à la métapsychologie. Lise Marceau nous propose une synthèse de la proposition d'une troisième topique, à partir de son élaboration plus récente par W. Reid, mais en référence aussi à ses racines dans la pensée de Winnicott. Puis, c'est sous l'angle de la première topique qu'André Jacques utilise la métaphore des zones humides dans l'environnement pour discuter de la fonction essentielle du préconscient, de même que de la crainte que cet entre-deux-zones peut inspirer. 